\title{
Adventures with CRYSTALS: developing methods and tools with an in-house refinement code
}

\author{
Richard I. Cooper
}

University of Oxford, Chemical Crystallography, Dept. Chemistry, Mansfield Rd, Oxford OX1 3TA, UK, richard.cooper@chem.ox.ac.uk

The software package CRYSTALS [1] is based on computer codes and algorithms developed in the Chemical Crystallography Laboratory in Oxford over almost 50 years. The program is still developed, tested and distributed and is used around the world to analyse structures from single crystal scattering experiments.

The source code of CRYSTALS is available in-house (and from github.com/ChemCryst/crystals) and this gives us the opportunity to both rapidly prototype, and give a stable home to, new structure analysis methods, without having to re-implement the core crystallographic supporting routines on every occasion.

Examples of recent developments facilitated by this availability of code will be presented, including:

- 'HUG and SQUEEZE' [2] - addition of resonant scattering effects to structure factor corrections from SQUEEZE [3] can enable determination of absolute structure even when the resonantly scattering atoms are present in the SQUEEZE'd void (e.g., dichloromethane solvent);

- a profusion of displacement parameter restraints (e.g., Fig. 1) for encouraging and enforcing physically meaningful atomic displacement distributions [4];

- an applied use of built-in statistical analysis of least squares leverage to identify inappropriate weighting of one or more restraints in a refinement.
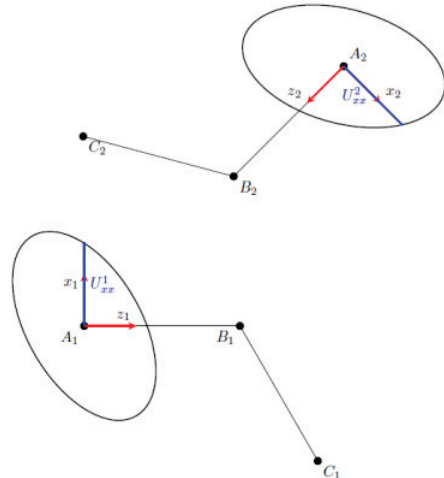

Fig. 1. ULIJ restraint: restrains all the elements of two ADPs to be equal, each in their own local coordinate system.

\section{References}

[1] Betteridge, P. W., Carruthers, J. R., Cooper, R. I., Prout, K., \& Watkin, D. J. (2003). J. Appl. Cryst., 36, 1487. [2] Cooper, R. I., Flack, H. D. \& Watkin, D. J. (2017). Acta Cryst. C73, 845-853. [3] Spek, A. L. (2015). Acta Cryst. C71, 9-18. [4] Parois, P., Arnold, J. \& Cooper, R. I. (2018). J Appl Cryst, 51, 1059-1068. 